\title{
MAIT cells: not just another brick in the wall
}

\author{
Karlijn Debusschere, ${ }^{1,2}$ Rik J Lories, ${ }^{3}$ Dirk Elewaut ${ }^{1,2}$
}

The link between gut and joint inflammation in spondyloarthritis $(\mathrm{SpA})$ is well established, in particular in ankylosing spondylitis (AS) and peripheral SpA. In 1995, Mielants et $a l^{1}$ discovered that almost $50 \%$ of patients with SpA have subclinical gut inflammation, of which a fraction develops Crohn's disease over time. Recent studies clearly showed that the onset of disease and disease severity are linked to the presence of subclinical gut inflammation. ${ }^{2}$ However, the mechanism behind this phenomenon is hitherto not fully elucidated. Over the past decade, the interleukin (IL)-23/IL-17-axis has been put forward as a key player in the pathogenesis of SpA. Polymorphisms in the IL23R gene were found both in SpA and in inflammatory bowel disease (IBD), ${ }^{3}$ linking pathology in these physically distant sites. Cells that are typically responsive to IL-23 are IL-23R ${ }^{+} \mathrm{ROR}_{\gamma \mathrm{t}^{+}}$ IL-17A-producing cells, such as $\mathrm{T}_{\mathrm{H}} 17$ cells. In multiple inflammatory diseases, such as multiple sclerosis (MS), IBD and $\mathrm{SpA}$, it is assumed that there is an overall distortion of the cytokine profile towards IL-17A, contributing to disease. ${ }^{4}$ However, it should be noted that in IBD IL-17A might also exert tissue-protective functions and that mechanisms driving gut inflammation in SpA do not necessarily represent those contributing to the development of full-blown IBD. ${ }^{3}$ Intriguingly, Sherlock et $a l^{5}$ showed that systemic IL-23 overexpression is able to drive the development of enthesitis via enthesis-resident CD $3{ }^{+} \mathrm{CD}^{-}{ }^{-} \mathrm{CD} 8{ }^{-} \mathrm{ROR}_{\gamma \mathrm{t}^{+}} \mathrm{IL}_{23 \mathrm{R}^{+}}$T cells, independently of $\mathrm{T}_{\mathrm{H}} 17$ cells. The concept that not $\mathrm{T}_{\mathrm{H}} 17$ cells but innate-like $\mathrm{T}$ cells such as $\gamma \delta$ T cells, invariant natural killer $\mathrm{T}$ cells (iNKT) and mucosal-associated invariant $\mathrm{T}$ (MAIT) cells are the main source of IL-17A recently gained support. ${ }^{6}$

Interestingly, innate-like $\mathrm{T}$ cells such as MAIT cells act at the intersection of the

\footnotetext{
${ }^{1}$ Department of Internal Medicine-Rheumatology, Ghent University, Ghent, Belgium; ${ }^{2}$ Unit of Molecular Immunology and Inflammation, Inflammation Research Center-VIB, Ghent, Belgium; ${ }^{3} \mathrm{KU}$ Leuven and Division of Rheumatology, Laboratory of Tissue Homeostasis and Disease, Skeletal Biology and Engineering Research Center, University Hospitals Leuven, Leuven, Belgium
}

Correspondence to Dr Dirk Elewaut, Department of Rheumatology, UZ Gent, OK12IB, De Pintelaan 185, Ghent 9000, Belgium; Dirk.elewaut@ugent.be innate and adaptive immune systems. ${ }^{6}$ They rapidly produce cytokines with a $\mathrm{T}_{\mathrm{H}} 1$ (tumour necrosis factor (TNF) and interferon-gamma (IFN $\gamma)$ ) or $\mathrm{T}_{\mathrm{H}} 17$ (IL-17A) profile upon $\mathrm{T}$ cell receptor (TCR)-dependent and TCR-independent signals. ${ }^{7}$ It is, thus, obvious that the activation of such potent cells incurs risks. ${ }^{8}$ Hence, multiple reports describe the involvement of MAIT cells in inflammatory diseases such as MS, IBD and rheumatoid arthritis (RA). ${ }^{4} \mathrm{MR} 1^{-/-}$mice, which lack MAIT cells, had reduced severity of antibody-induced arthritis and collagen-induced arthritis, both models for RA, suggesting a pathological role for MAIT cells in RA. ${ }^{9}$ In this issue of Annals of the Rheumatic diseases, Gracey et al ${ }^{10}$ are the first to investigate the number of MAIT cells and their functional phenotype in patients with AS. As in many other inflammatory diseases, ${ }^{4}$ they report a relative abundance of IL-17-producing MAIT cells in patients with AS, both systemically and in synovial fluid compared with healthy controls and patients with RA. ${ }^{10}$ However, it still needs to be elucidated to what extent these cells are developmentally programmed to attain this IL-17A-producing phenotype or if they acquire these characteristics in the periphery under inflammatory conditions after being fully matured. Furthermore, it should be noted that the majority of MAIT cells (80-90\%) produce TNF and IFN $\gamma$ compared with approximately $3 \%$ IL-17A-producing MAIT cells. Despite their overall low numbers, they might still be critical in driving disease as their numbers are heightened both systemically and in synovial fluid of patients with AS compared with healthy controls and patients with RA. ${ }^{10}$

A key question is whether these cells can be modulated under inflammatory conditions towards a tissue-protective phenotype. ${ }^{6}$ Modulation of MAIT cells might be a more interesting therapeutic approach than modulation of their phenotypical and functional counterparts, the iNKT cells. MAIT cell numbers are relatively high in humans $(1-10 \%$ of circulating T cells, $20-45 \%$ of T cells in the liver and $3-5 \%$ of lymphoid cells in the intestinal mucosa), compared with numbers of iNKT cells. The opposite situation is present in mice, where iNKT cells are rather abundant compared with MAIT cells, the latter also not displaying a functionally mature phenotype as can be found in humans. ${ }^{7}$ This bias might suggest an evolutionary need for one or the other cell type. ${ }^{11}$ In many inflammatory diseases, systemic MAIT cell numbers are lowered compared with healthy controls, with a relative enrichment in the inflamed tissues. ${ }^{4}$ A similar situation was found in patients with AS and patients with RA, where MAIT cells were enriched in the synovial fluid and lowered systemically. Even though the numbers between AS and RA seem comparable, they phenotypically differ as only in patients with AS MAIT cells had a predominant IL-17A profile. ${ }^{10}$ Human MAIT cells are often characterised as $\mathrm{CD}^{+}$TCRV $\alpha 7.2^{+}$ CD161 ${ }^{\text {hi }}$ T cells. ${ }^{4} 12$ The use of this marker panel is, however, not without any risks, especially when used to characterise MAIT cells in patients suffering from inflammatory diseases. Upon activation, MAIT cells downregulate CD161 and the expression of this marker can be confounded by the use of corticosteroids. ${ }^{4}$ In axial SpA and, to a lesser extent, peripheral $\mathrm{SpA}$, corticosteroids are not the therapy of choice $^{13}$ and decreases in MAIT cell frequencies due to corticosteroid use are not to be expected. ${ }^{14}$ The results described by Gracey et al ${ }^{10}$ come from a cross-sectional observational cohort, providing very useful insight into MAIT cell functionality in AS. However, the patients have been exposed to a wide variety of treatment strategies, possibly influencing data outcome. It would thus be interesting to see if similar results could be obtained in patients with newonset, therapy-naïve SpA.

What makes MAIT cells an even more appealing cell type in the light of combined gut-joint disease, is that they typically reside in the epithelium and lamina propria of the gut, a site of important host-microbial interplay. ${ }^{15}$ Here, they fully mature after thymic emigration, a process that depends on the presence of the commensal microbial flora and $\mathrm{B}$ cells. ${ }^{16} \mathrm{~A}$ hallmark of these cells is their highly restricted TCR V $\alpha$ repertoire. ${ }^{7}$ They react to non-peptide antigens, bound by the non-classical class I antigenpresenting molecule, MR1 that is highly conserved across mammalian species (figure 1). MR1 presents vitamin $\mathrm{B}_{2}$ metabolites, which are specific to certain species of the microbiota such as Salmonella enterica serovar Typhimurium, to MAIT cells. ${ }^{72}$ However, the exact set of antigens recognised by MAIT cells is 


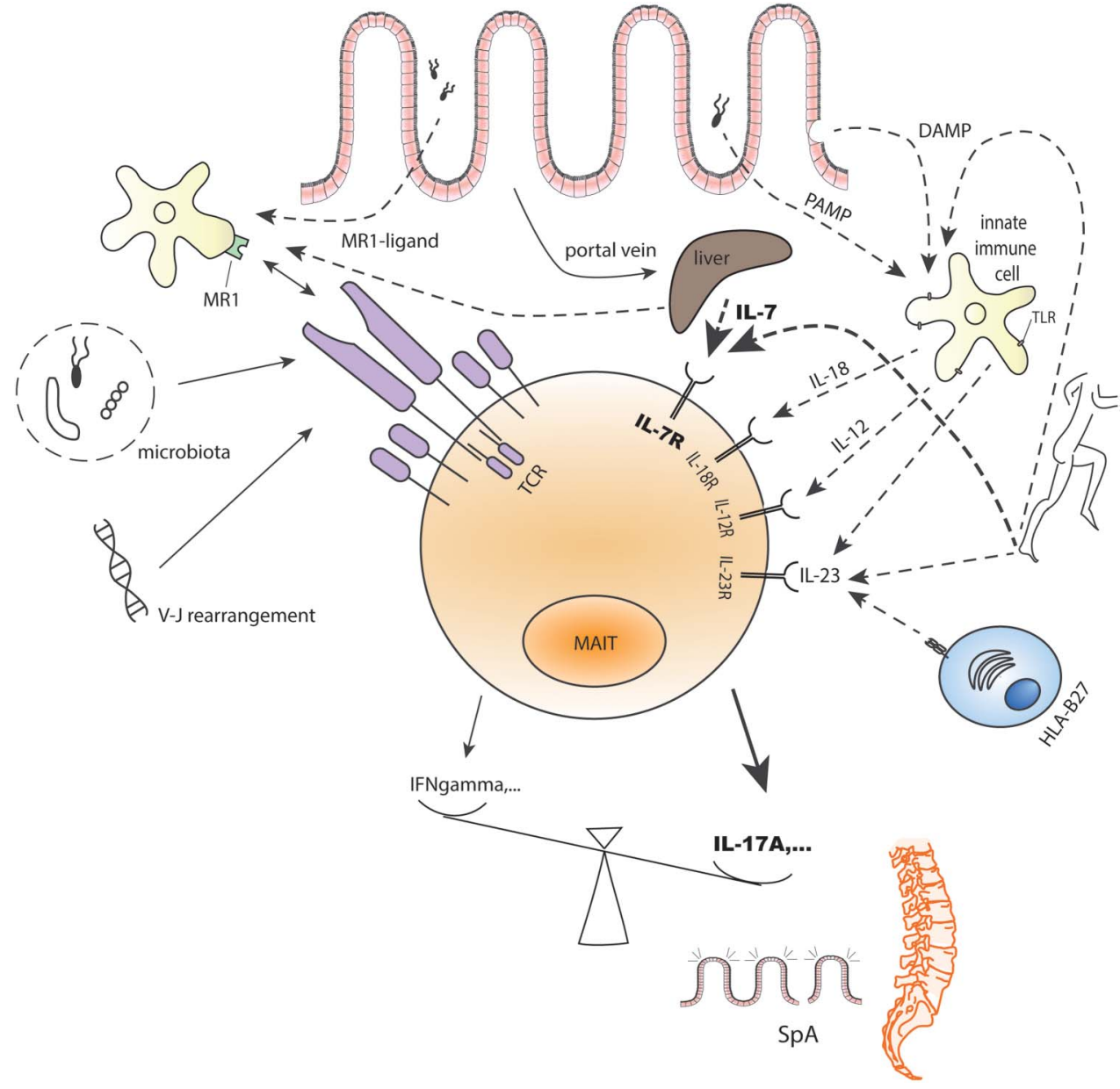

Figure 1 Conceptual framework of the role of mucosal-associated invariant T (MAIT) cells in spondyloarthritis (SpA) -MAIT cells express a semi-invariant T cell receptor (TCR), as well as high levels of interleukin (IL)-7R, IL-18R, IL-12R and IL-23R. They can be activated in a TCR-dependent and TCR-independent manner. TCR-dependent activation is via antigen recognition of vitamin B derivatives, by-products from the microbiome, that are presented via the non-classical class I antigen-presenting molecule MR1. These antigens can be picked up directly at the mucosal border or can be transported via the portal vein to the liver, where many MAIT cells can be found. TCR rearrangement is determined by genetic factors and will influence antigen recognition by MAIT cells. Furthermore, maturation of MAIT cells depends on the presence of commensal microbial flora and thus likely TCR signalling. TCR-independent activation of MAIT cells is cytokine induced, directly or after activation of innate immune cells via their toll-like receptors (TLRs). These TLRs can bind both pathogen-associated molecular patterns (PAMPs) from the gut as well as danger-associated molecular patterns (DAMPs) released upon cellular stress (epithelial damage, biomechanical stress, human leucocyte antigen (HLA)-B27 misfolding resulting in unfolded protein response). Furthermore, TLR signalling in the liver as well as stress in synovial fibroblasts can lead to IL-7 production, which solely or in combination with anti-CD3/CD28 can lead to MAIT cell activation. All these signals eventually result in the activation of MAIT cells and consequent production of pro-inflammatory cytokines, such as interferon-gamma (IFN $\gamma$ ) and IL-17A. The equilibrium between these cytokines is invaluable in the pathogenesis of SpA, tipping the balance towards resolution of inflammation or full-blown chronic disease (gut inflammation and ankylosis of the spine), the latter is hypothesised to happen under high IL-17A levels.

not yet fully known and it is possible that MAIT cell TCR usage differs between individuals. ${ }^{7}$ Given the antigen restriction that is typical for MAIT cells due to the expression of a semi-invariant TCR, their typical effector memory phenotypeallowing rapid production of large amounts of cytokines without prior clonal expansion-and their presence in the gut lining, they may function as first responders towards aberrant microbial signals but may also sustain abnormal inflammatory reactions. Genetic factors underlying the, however limited, divergence in TCR usage towards gut-derived antigens might partly form the basis towards aberrant immune reactions due to TCR signalling as seen in autoinflammatory diseases such as SpA (figure 1). However, Gracey et al ${ }^{10}$ showed that TCR-activated reaction in patients with AS was not different than that in healthy controls.

The results obtained by Gracey et al, ${ }^{10}$ suggest that TCR-independent mechanisms might be more important in driving disease in SpA than TCR-dependent mechanisms. In this study, they used Salmonella enterica spp to activate MAIT cells, but as mentioned by the authors, the exact nature of the Salmonella antigen in this assay is unknown. Other known MR1 antigens, however, have not been tested. TCR-independent activation of MAIT cells can be induced via cytokines secreted by innate cells upon toll-like receptor (TLR) signalling with pathogen and/or dangerassociated molecular patterns (PAMPs resp. DAMPs). ${ }^{7}$ DAMPs might be released upon human leucocyte antigen (HLA)-B27 misfolding, ${ }^{6}$ oxygen stress ${ }^{17}$ and upon biomechanical stress, ${ }^{18}$ contributing to the pathogenesis of SpA (figure 1). Of interest is that these DAMPs can occur both in the gut and in the joint, linking these physically distant sites. A combination of IL-18 and IL-12 can steer MAIT cells to produce large amounts of IFN $\gamma^{7}$ and IL-23 (with or without IL-1 $\beta$ ) is also used to activate MAIT cells. ${ }^{19}$ Gracey et al, ${ }^{10}$ however, looked into the effect of IL-7 stimulation on these cells because of the presence of 
IL-7R single nucleotide polymorphisms linked to AS, high expression pattern of the IL-7R on MAIT cells and heightened IL-7 levels in serum of patients with AS. IL-7 is typically produced by stromal cells, such as fibroblast-like synoviocytes ${ }^{20}$ and the liver can also be a primary source of IL-7 upon TLR signalling, ${ }^{21}$ which is interesting because $80 \%$ of blood flow through the liver comes from the portal vein and thus the gut (figure 1). ${ }^{22}$ Here it was shown that IL-7 could induce an IL-17A profile in MAIT cells that was different between patients with AS and healthy controls. Furthermore, activation with IL-23 also had no effect on MAIT cell cytokine profile. $^{10}$

The results of the paper by Gracey et $a l^{10}$ thus shed new light on the involvement of MAIT cells in SpA, more specifically AS. Food for thought is the finding that TCR-independent mechanisms may be more important in shaping MAIT cells than TCR-dependent mechanisms. In this paper, we may assume that their role in disease initiation and perpetuation will more likely depend on their capacity to rapidly produce $\mathrm{T}$ cell-related cytokines in an inflammatory setting, where the ruling cytokine environment will direct MAIT cell functionality. However, their TCR dependency in AS may depend on the nature of the antigen encountered and studies involving the fully known spectrum of MR1 ligands will thus be indispensable to answer this question. Furthermore, since MAIT cells depend on the microbial flora for their maturation, TCR signalling might also play a role early on in life during this maturation process. All in all, MAIT cells are not just another brick in the wall and future research into this cell population will give new insight into the pathogenesis of SpA.

Funding Collaborative research of the authors (RJL and DE) on the topic is supported by grant G.0946.14 from the Research Foundation-Flanders (FWO) and by the DevRepair (P7/07) IUAP-VII Network (Belgian Federal Government). DE is supported by a fund of
Research Foundation-Flanders (FWO) and the Research Council of Ghent University. DE is also a member of a multidisciplinary research platform (MRP) of Ghent University. DE received funding from the EU's seventh framework programme under EC-GA no. 305266 'MIAMI'. KD is supported by a grant of Research Foundation-Flanders (FWO) (FW015/ASP/ 051).

Competing interests None declared.

Provenance and peer review Commissioned; externally peer reviewed.

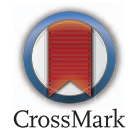

To cite Debusschere K, Lories RJ, Elewaut D. Ann Rheum Dis 2016;75:2057-2059.

Received 5 June 2016

Revised 5 July 2016

Accepted 9 July 2016

Published Online First 29 July 2016

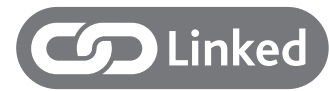

- http://dx.doi.org/10.1136/annrheumdis-2015208902

Ann Rheum Dis 2016;75:2057-2059.

doi:10.1136/annrheumdis-2016-209695

\section{REFERENCES}

1 Mielants H, Veys EM, Cuvelier C, et al. The evolution of spondyloarthropathies in relation to gut histology. II. Histological aspects. J Rheumatol 1995;22:2273-8.

2 Van Praet L, Jans L, Carron P, et al. Degree of bone marrow oedema in sacroiliac joints of patients with axial spondyloarthritis is linked to gut inflammation and Male sex: results from the GIANT cohort. Ann Rheum Dis 2014;73:1186-9.

3 De Wilde K, Debusschere K, Beeckman S, et al. Integrating the pathogenesis of spondyloarthritis: gut and joint united? Curr Opin Rheumatol 2015:27:189-96.

4 Hinks TS. Mucosal-associated invariant T cells in autoimmunity, immune-mediated diseases and airways disease. Immunology 2016;148:1-12.

5 Sherlock JP, Joyce-Shaikh B, Turner SP, et al. IL-23 induces spondyloarthropathy by acting on ROR- $\gamma \mathrm{t}+$ CD3+CD4-CD8- entheseal resident T cells. Nat Med 2012;18:1069-76.

6 Venken K, Elewaut D. IL-23 responsive innate-like T cells in spondyloarthritis: the less frequent they are, the more vital they appear. Curr Rheumatol Rep 2015;17:30.
7 Chandra S, Kronenberg M. Activation and Function of iNKT and MAIT Cells. Adv Immunol 2015;127:145-201.

8 Wencker M, Turchinovich G, Di Marco Barros R, et al. Innate-like T cells straddle innate and adaptive immunity by altering antigen-receptor responsiveness Nat Immunol 2014;15:80-7.

9 Chiba A, Tajima R, Tomi C, et al. Mucosal-associated invariant $T$ cells promote inflammation and exacerbate disease in murine models of arthritis. Arthritis Rheum 2012:64:153-61.

10 Gracey E, Qaiyum Z, Almaghlouth I, et al. IL-7 primes IL-17 in mucosal-associated invariant T (MAIT) cells, which contribute to the Th17-axis in ankylosing spondylitis. Ann Rheum Dis 2016;75: 2123-31.

11 Treiner E, Duban L, Moura IC, et al. Mucosal-associated invariant T (MAIT) cells: an evolutionarily conserved T cell subset. Microbes Infect 2005; 7:552-9.

12 Le Bourhis L, Guerri L, Dusseaux M, et al. Mucosal-associated invariant T cells: unconventional development and function. Trends Immunol 2011:32:212-18.

13 Braun J, van den Berg R, Baraliakos X, et al. 2010 update of the ASAS/EULAR recommendations for the management of ankylosing spondylitis. Ann Rheum Dis 2011:70:896-904.

14 Cho YN, Kee SJ, Kim TJ, et al. Mucosal-associated invariant T cell deficiency in systemic lupus erythematosus. J Immunol 2014;193:3891-901.

15 Mowat AM, Agace WW. Regional specialization within the intestinal immune system. Nat Rev Immunol 2014;14:667-85.

16 Treiner E, Duban L, Bahram S, et al. Selection of evolutionarily conserved mucosal-associated invariant T cells by MR1. Nature 2003:422:164-9.

17 Park SY, Lee SW, Kim HY, et al. HMGB1 induces angiogenesis in rheumatoid arthritis via HIF-1 $\alpha$ activation. Eur J Immunol 2015;45:1216-27.

18 Jacques $P$, Lambrecht $S$, Verheugen $E$, et al. Proof of concept: enthesitis and new bone formation in spondyloarthritis are driven by mechanical strain and stromal cells. Ann Rheum Dis 2014;73:437-45.

19 Tang XZ, Jo J, Tan AT, et al. IL-7 licenses activation of human liver intrasinusoidal mucosal-associated invariant T cells. J Immunol 2013;190: 3142-52.

20 Harada S, Yamamura M, Okamoto $\mathrm{H}$, et al. Production of interleukin-7 and interleukin- 15 by fibroblast-like synoviocytes from patients with rheumatoid arthritis. Arthritis Rheum 1999;42:1508-16.

21 Sawa $Y$, Arima $Y$, Ogura $H$, et al. Hepatic interleukin-7 expression regulates $\mathrm{T}$ cell responses. Immunity 2009;30:447-57.

22 Robinson MW, Harmon C, O'Farrelly C. Liver immunology and its role in inflammation and homeostasis. Cell Mol Immunol 2016;13:267-76. 